\title{
Macromolecular Model Building Over the Web
}

\author{
Egor Sobolev', Philipp Heuser², Victor S. Lamzin ${ }^{3}$ \\ ${ }^{I}$ European XFEL GmbH, Holzkoppel 4, 22869 Schenefeld, Germany \\ ${ }^{2}$ Helmholtz Imaging Platform, DESY, Notkestrasse 85,22607 Hamburg, Germany. \\ ${ }^{3}$ European Molecular Biology Laboratory, c/o DESY, Notkestrasse 85, 22607 Hamburg, Germany \\ victor@embl-hamburg.de
}

The ARP/wARP software provides automated model building in macromolecular crystallography and cryo-EM maps for structures of proteins, and their complexes with nucleic acids and small-molecule ligands. The ARP/wARP remote service for macromolecular model building has been available since 2004 and was used to provide tens of thousands of model-building jobs remotely submitted by more than 4,000 users. A comprehensive description of the ARP/wARP web service, including a historical perspective, will be provided. To allow the user a direct monitoring of the model-building task, its progress and accumulated results (including the Wilson plot, the development of crystallographic R/Rfree-factors, the number of residues built) are displayed graphically and in a tabular form as well as JavaScript-based cartoons of the built structures. The output files can also be downloaded when the job is completed. A user can rerun jobs with modified parameters and the results of these can be compared to each other. The analysis of the accumulated data and a number of take-home messages will be presented.

Keywords: ARP/wARP, web service, model building, macromolecular crystallography, cryo-electron microscopy 